\title{
PROPOSTA PARA O ALINHAMENTO ENTRE O PLANO ESTRATÉGICO INSTITUCIONAL DE UM LABORATÓRIO FARMACÊUTICO OFICIAL E O DEPARTAMENTO DE TI
}

\author{
PROPOSAL FOR THE ALIGNMENT BETWEEN THE INSTITUTIONAL \\ STRATEGIC PLAN OF AN OFFICIAL PHARMACEUTICAL \\ LABORATORY AND THE IT DEPARTMENT
}

Saulo Moura da Silva ${ }^{1}$

Tatiana Aragao Figueiredo ${ }^{2}$

Jorge Lima de Magalhaes ${ }^{3}$

\begin{abstract}
Resumo: As organizações, quer sejam públicas ou privadas, necessitam de estratégias bem definidas para seus negócios, bem como um plano de gestão de seus recursos. Para o setor farmacêutico, não é diferente, haja vista que se trata de um setor que movimenta US\$1,3 trilhões anualmente Quintiles and IMS Health, Inc. [IQVIA] (2019). Os Laboratórios Farmacêuticos Oficias (LFO) entram nesse contexto, com maior responsabilidade, pois gerenciam recursos públicos como devolutiva à Sociedade de medicamentos negligenciados pelas empresas farmacêuticas privadas. Contudo, a estratégia também deve estar alinhada com os demais setores da corporação, já que é sabido que falta de alinhamento estratégico em empresas acaba fazendo com que muitos recursos humanos e financeiros sejam empregados em projetos e iniciativas não tão relevantes para o negócio. Nesse sentido, a Tecnologia da Informação (TI) deve estar não só nas corporações, como também alinhada ao negócio de fato. Na busca pelo alinhamento entre a área de TI e o núcleo do negócio de um LFO, este trabalho pretende propor um modelo de alinhamento estratégico, tomando por base pontos dos modelos de Broadbeck e Hoppen (2003) e Kearns e Sabherwal (2006) estruturados sob a ideia trazida pelo modelo sistemático de Moreira (2002). Como resultado, uma sistemática para o alinhamento foi montada, assim como aplicada de maneira parcial no LFO em questão. O presente trabalho, portanto, além de contribuir diretamente para a estratégia organizacional do LFO, também contribui socialmente para todas as demais empresas ou setores públicos que desejarem aplicar o método para otimizar seus processos e práticas gerenciais e estratégicas.
\end{abstract}

Palavras-chave: Gerenciamento estratégico. Gestão organizacional. Gestão do conhecimento. Indústria farmacêutica.

Abstract: Organizations, whether public or private, need a well-defined strategy for their business as well as a resource management plan. It is no different for the pharmaceutical sector, considering a sector that moves US \$ 1.3 trillion annually Quintiles and IMS Health, Inc. [IQVIA] (2019) The Official Pharmaceutical Laboratories (LFO) enter into this context, with greater responsibility, as they manage public resources as a return to the Society of medicines neglected by private pharmaceutical companies. However, the strategy must also be aligned with the other sectors of the corporation, since it is known that the lack of strategic alignment in companies ends up causing many human and financial resources to be used in projects and initiatives that are not so relevant to the business. In this sense, with Information Technology (IT) being increasingly permeated in corporations, it is necessary that it is also aligned with the business. In the search for alignment between the IT area and the core of an LFO's business, this work intends to propose a strategic alignment model, based on points of the Broadbeck and Hoppen (2003) and Kearns and Sabherwal (2006) models structured under the idea brought by Moreira's systematic model (2002). As a result, a system for alignment was put in place, as well as partially applied to the LFO in question. The work in question, in addition to contributing directly to the organizational strategy of the LFO, also contributes socially to all other companies or public sectors that wish to apply the method to optimize their managerial and strategic processes and practices.

Keywords: Strategic management. Organizational management. Knowledge management. Pharmaceutical industry.

${ }^{1}$ Programa de Pós-Graduação em Gestão, Pesquisa e Desenvolvimento na Indústria Farmacêutica. Farmanguinhos - Fundação Oswaldo Cruz, Rio de Janeiro, RJ - Brasil; saulo.silva@far.fiocruz.br

(D) 2 Programa de Pós-Graduação em Gestão, Pesquisa e Desenvolvimento na Indústria Farmacêutica. Farmanguinhos - Fundação Oswaldo Cruz, Rio de Janeiro, RJ e Médicos Sem Fronteiras; tatiana.figueiredo@far.fiocruz.br

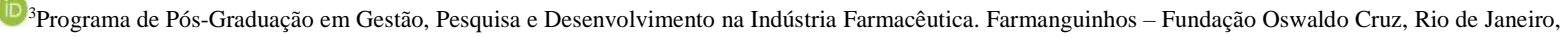
RJ e Global Health and Tropical Medicine, Instituto de Higiene e Medicina Tropical, Universidade NOVA de Lisboa; jorge.magalhaes@ far.fiocruz.br

\section{Cite como}

American Psychological Association (APA)

Silva, S. M., Figueiredo, T. A., \& Magalhaes, J. L. (2020, jul./dez.). Proposta para o alinhamento entre o plano estratégico institucional de um laboratório farmacêutico oficial e o departamento de TI. Revista Inovação, Projetos e Tecnologias, São Paulo, 8(2), 198-220. https://doi.org/10.5585/iptec.v8i2.18660. 


\section{Introdução}

O Sistema Único de Saúde (SUS) teve sua criação no início dos anos 90. A nova Constituição Federal de 1988 colocou a saúde como direito de todos e dever do Estado. Nesse contexto, uma das formas de promoção, proteção e recuperação da saúde é a Assistência Farmacêutica. Ela tem como ponto central o acesso aos medicamentos, principalmente àqueles considerados essenciais. Esse acesso pode ser proporcionado através de várias ações intersetoriais no mercado, como também pela formulação de medicamentos pelos laboratórios farmacêuticos privados e públicos (Magalhães, 2010a; Mendes et al., 2018).

A Política Nacional de Medicamentos (PNM), estabelecida em 1998, visa a garantia da segurança, eficácia e qualidade dos medicamentos, a promoção do uso racional e o acesso da população àqueles considerados essenciais. Tem, assim, a produção pública de medicamentos como um de seus objetivos (Vasconcelos et al., 2017). Posteriormente, no ano de 2004, o governo brasileiro inseriu a indústria farmacêutica como atividade-chave para o desenvolvimento do país e selecionou a indústria de fármacos e medicamentos como setor estratégico da política industrial. Com isso, o país passou a formular políticas e ações verticais para tal setor, através do envolvimento de órgãos de governo, associações de classe e empresas (Palmeira et al., 2012).

Em 2019, o Ministério da Saúde (MS) do Brasil conta com 21 Laboratórios Farmacêuticos Oficiais (LFO). Estes são vinculados aos governos estaduais e federal. Eles estão dispersos em todo território nacional devido à sua grandiosidade, diversidade de nossa população e condições de saúde populacionais. A capacidade instalada existente produz cerca de $30 \%$ dos medicamentos utilizados pelo SUS (Brasil, 2019).

Ao mesmo tempo em que as empresas farmacêuticas privadas preocupam-se com as doenças crônicas e cardiovasculares, foco da atenção internacional, nota-se que as enfermidades infecciosas e as negligenciadas (aquelas relacionadas à pobreza) não provocam muito interesse do mercado privado por não gerarem altos lucros. Nesse sentido, uma forma de atuação dos LFO é atender as demandas do Ministério da Saúde, bem como das secretarias de saúde, para aqueles produtos que não são interessantes para indústria privada, principalmente em relação às doenças negligenciadas (Figueiredo, 2015; Margotto 2019). Para atender essas necessidades em relação à atenção à saúde, o governo brasileiro passou a realizar ações de modo a fomentar a produção pública de medicamentos, com a finalidade de diminuir a dependência do país. Os LFO também atuariam na manufatura e na estratégia indutora de políticas, desenvolvimento de 
formulações e novos fármacos bem como na regulação de preços no mercado nacional (Magalhães et al., 2011; Pepe, 2016; Mori, 2019).

Apesar da relevância dos LFO, para fazer parte de um negócio altamente dinâmico como o setor farmacêutico, eles têm de lidar com a velocidade das transformações e seus impactos nos processos de produção e entrega para o SUS. Uma forma de atingir essas necessidades seria através da execução do Planejamento Estratégico (PE). Por permitir que diversas ferramentas estratégicas sejam aplicadas durante suas etapas, este mecanismo de gestão é muito utilizado pelas organizações, cujo objetivo é a integração e articulação das diversas áreas da empresa, de forma a guiá-las para um mesmo caminho e permitir resposta às rápidas mudanças no ambiente (Valente \& Brasil, 2019). O processo de alinhamento estratégico é de suma importância na condução das atividades de uma empresa e no contexto dos LFO não seria diferente. A falta deste alinhamento dentro das empresas acaba fazendo com que muitos recursos humanos e financeiros sejam empregados em projetos e iniciativas que não seriam as mais relevantes para o negócio.

Segundo Kaplan e Norton (2006), várias empresas se comportam como barcos sem coordenação. Possuem diversas unidades de negócio eficientes, com executivos muito capacitados, motivados e experientes, mas não possuem alinhamento entre seus esforços. Esses mesmos autores identificaram que, ao classificar as práticas de gestão de empresa que utilizavam scorecards, nos cinco princípios para a implementação bem-sucedida da estratégia (mobilização, tradução da estratégia, alinhamento da organização, motivação dos empregados e gerenciamento). As empresas que obtiveram benefícios expressivos com o uso do BSC (Balanced Scoredcard) foram as que melhor aplicaram a etapa de alinhamento organizacional (Carvalho, 2017).

O Alinhamento Estratégico entre as áreas de negócio de uma empresa é de suma importância para o desempenho da mesma no mercado. Considerando que o mundo está cada vez mais conectado e dependente das tecnologias da informação, se torna urgente obter diferencial competitivo entre os demais pares produtivos, bem como um alinhamento entre as estratégias institucionais e de tecnologia da informação. Isso pode ser considerado como imperativo a fim de garantir melhorias, tais como eficiência, eficácia, economicidade e fluidez entre os fluxos de trabalho.

A indústria farmacêutica, seja privada ou pública, é de grande relevância para a saúde pública, portanto, requer estratégia organizacional na gestão de recursos, a fim de atender as necessidades de seus clientes, no setor público, o Sistema Único de Saúde. Neste sentido, este trabalho pretende propor um modelo de alinhamento estratégico entre a área de Tecnologia da 
Informação e o núcleo do negócio de um LFO, bem como a aplicação parcial do modelo proposto.

\section{Referencial teórico}

\subsection{Política industrial e laboratórios farmacêuticos oficiais}

Indo ao encontro do que é preconizado pela PNM, a fim de tentar minimizar as dificuldades existentes no país para lidar com as questões relativas à pesquisa, ao desenvolvimento produtivo e à inovação tecnológica na área farmacêutica, o Complexo Econômico-Industrial da Saúde (CEIS) trouxe, com um olhar sistêmico, a necessidade de maior nacionalização da cadeia produtiva, de estratégias de inovação mais robustas, de melhorias para as políticas de estímulo à pesquisa e inovação de segmentos relacionados às doenças negligenciadas, além da implementação de ações transversais de suporte tecnológico. Essas questões seriam as mais impactantes para a pouca inovação nessa indústria (Figueiredo, 2015; Gadelha et al., 2010; Bermudez et al., 2018).

Nesse sentido, em 2007, o CEIS foi introduzido como eixo estratégico para o desenvolvimento produtivo e de inovação, através do Programa Mais Saúde (PAC da Saúde). Seguindo essa mesma linha, em 2008 o CEIS foi reconhecido, através da publicação da Política de Desenvolvimento Produtivo (PDP), como uma das seis áreas estratégicas para o desenvolvimento nacional (Silva, 2014).

Essa nova política trouxe consigo o fomento ao desenvolvimento interno da indústria nacional de fármacos de base química ou biotecnológica (tanto produtivo quanto no que diz respeito à inovação), medicamentos de alta complexidade tecnológica e produtos de saúde. Associando isso a estratégia de transferência de conhecimento por meio de tecnologias, acabou por ampliar o papel regulador dos Laboratórios Farmacêuticos Oficiais no mercado nacional (Rezende, 2013; Ticom, 2019).

Podemos observar que os LFO, em um contexto macro, seguem diretrizes superiores para a realização de suas funções, que são primordiais para a sociedade (Magalhães, 2010; Silva 2012). Tais questões colocam esses laboratórios como parte importante dentro da estratégia governamental adotada. Nesse sentido, se torna cada vez mais necessária a discussão e a implementação de ferramentas que possibilitem o alinhamento entre os atores envolvidos para que seu importante papel, dentro do sistema de saúde, seja cumprido de maneira satisfatória (Margotto, 2019). 


\subsection{Estratégia nas corporações e planejamento estratégico}

A estratégia funciona como uma estrutura que baliza as escolhas e acaba determinando a natureza e o rumo de uma organização. Ela é fundamental, pois possibilita a geração de um diferencial competitivo para a empresa. Dentro desse contexto, e considerando que o desenvolvimento de uma estratégia engloba elementos racionais e não racionais, podemos transformar o planejamento estratégico e a gestão estratégica em ferramentas para guiar o desenvolvimento estratégico em uma corporação (Gonçalves et al., 2012). Segundo Porter (2008), todas as organizações possuem uma estratégia competitiva, implícita ou explícita que pode estar formalizada ou não. A estratégia competitiva busca a maximização do diferencial de negócio de uma empresa, em relação aos seus concorrentes, e, para isso, estabelece as formas de competição desta empresa, bem como suas políticas e metas necessárias para alcançar seus objetivos.

Mintzberg et al. (2010) ampliaram o conceito de estratégia, através da separação dos entendimentos que se podem ter dentro desse conceito. Eles dividem estratégia em: algo deliberado; planos e padrões; posição e perspectiva; e, por fim, truque. Já para Prahalad e Hamel (1997), a principal estratégia vem de dentro da organização, através da sua competência essencial. Essa competência, formadora de vantagem competitiva sustentável, é colocada pelos autores como a capacidade de se aprender coletivamente na organização, em especial no que diz respeito à forma de coordenar as mais diferentes habilidades de produção e integrar as multíplices correntes de tecnologia.

Hitt, Ireland e Hoskisson (2019) seguem Prahalad e Hamel (1997b) ao definir estratégia como um conjunto coeso de acordos e ações firmadas em uma organização, que exploram as competências essenciais desta a fim de obter vantagem competitiva. Nesse sentido, a utilização de uma estratégia diferenciada permite à organização alcançar vantagem competitiva sobre seus concorrentes. Já Teeratansirikool et al. (2013) trazem evidências sobre como a utilização de estratégias auxilia na obtenção de vantagens competitivas, acarretando melhorias para o desempenho das organizações.

Portanto, os diversos sentidos dados para "estratégia" se complementam e faz-se necessária a transformação das ideias em algo mais palpável para as corporações. Assim, a utilização de ferramentas estratégicas, tais quais o PE e a gestão estratégica citadas por Gonçalves e Reis Neto (2006), pode ajudar na aplicabilidade e no desenvolvimento das estratégias nas corporações. 
O Planejamento Estratégico (PE) é um procedimento gerencial que permite estabelecer o caminho a ser tomado pelas empresas. Este procedimento busca uma interação mais assertiva com o ambiente, considerando a habilidade da empresa para assimilar as adequações necessárias. Ressalta-se que o PE é mais amplo, compreendendo toda a organização; ele é a base do planejamento organizacional. Portanto, um bom planejamento é parte essencial de uma boa administração (Dias, 2015; Valente \& Brasil, 2019).

Historicamente o PE apresenta-se como mais do que uma mera ferramenta ou técnica formal, ele pode ser tomado como uma forma de gerir as organizações e a interação entre essa forma e os gestores. Alguns autores colocam que seu início ocorreu na década de 60, com a publicação de um artigo por Gilmore e Brandenburg na Harvad Bussiness Review. Ao mesmo tempo que outros ligam essa época com o marco de dez anos do início do Planejamento de longo prazo, sendo resposta à saturação e à decadência de grandes grupos à época (Dias, 2015; Souza et al., 2015).

O planejamento não deve ser visto apenas como uma prerrogativa da área de negócios, pois está cada vez mais ligado às tecnologias que o sustentam. Assim, seu alinhamento se torna premente nas organizações e, especialmente na área de TI, existe o Planejamento Estratégico de Tecnologia da Informação. O PE de TI (PETI) traz consigo um conjunto de metas estabelecidas para longo prazo, que tratam da infraestrutura e das principais iniciativas de TI que seriam imprescindíveis para que a organização pudesse alcançar as metas traçadas para o negócio (Dias, 2018).

\subsection{Planejamento estratégico de TI na administração pública}

Para gerenciar de maneira estratégica a tecnologia da informação, faz-se necessário, cada vez mais, o entendimento de como o papel dos sistemas de informação se transformou nos últimos tempos. Atualmente, tornou-se corriqueira a disseminação das informações através da internet utilizando-se ferramentas como sites, WhatsApp, Instagram dentre outras. Além disso, também faz parte da tecnologia da informação toda parte de infraestrutura física (hardware), lógica (software), além das redes de telecomunicações. São objetos tangíveis (servidores, PCs, roteadores e cabos de rede) e intangíveis (softwares diversos). Nesse contexto, a TI será utilizada para facilitar a aquisição, o processamento, o armazenamento, a transmissão e o compartilhamento de informações e conteúdos digitais (Arif, 2020).

Anwar Aini e Suryani (2019) apontam que a montagem de um planejamento estratégico de TI não deveria ter apenas foco na análise tecnológica, pois a conexão entre o negócio e a 
tecnologia é o que geraria lucros para as corporações. Tais conexões permitiriam que problemas advindos do negócio pudessem ser solucionados através de sistemas de informação, agregando valor ao mesmo. As estratégias voltadas para Tecnologia da Informação devem oferecer o suporte estrutural necessário para solucionar as necessidades de negócio das empresas.

Por isso, as organizações definem e orientam suas estratégias de negócio através do seu PE, sendo cada vez mais importante a participação e utilização de TI dentro das organizações. Nesse sentido, torna-se de suma importância o desenvolvimento de um Planejamento Estratégico de Tecnologia da Informação (PETI). As empresas que possuem o PETI podem obter vantagens competitivas, pois este ajudará na determinação dos novos Sistemas de Informação e melhorias nos existentes, a fim de suportar as estratégias traçadas para o negócio (Falsarella \& Jannuzzi, 2017). Em complemento, Magalhães et al. ( 2017) afirmam que o PETI funciona como um direcionador dos recursos de TI, tanto para área de pessoas, quanto para parte de sistemas e infraestrutura, estando esse direcionamento em consonância com as necessidades apontadas pela estratégia de negócio.

Assim como acontece com outras ferramentas de planejamento, que visam melhorar eficiência e eficácia nas empresas e são utilizadas pelo Estado, o planejamento relacionado à Tecnologia da Informação faz parte do conjunto de instrumentos aplicados pela administração pública. Sendo o PETI uma ferramenta para alinhamento de estratégias entre a TI e o negócio, ele não deve estar alheio a regra de planejamento preconizada pelo Estado. A Carta Magna de 1988, em seu artigo 174 imputa a obrigação legal de planejamento: "Art.174. Como procurador normativo e regulador da atividade econômica, o Estado desempenhará, na forma da lei, as funções de fiscalização, incentivo e planejamento, estando este definitivo para o setor público e indicativo para o setor privado".

Segundo Paranaiba e Foina (2017), na administração pública federal, a forma de ação não diverge do esperado para os demais setores. A TI é responsável por apoiar adequadamente as demandas dos órgãos visando um melhor atendimento da população que carece de celeridade, eficiência e inovação nos serviços públicos. Para isso, o instrumento Planejamento Estratégico de Tecnologia de Informação (PETI), é definido no Guia de Elaboração do PDTI do SISP - Sistema de Administração dos Recursos de Informação e Informática - como "um documento que complementa o Planejamento Estratégico Institucional, por meio do planejamento de sistemas de informação, conhecimentos e informática, possibilitando a definição de objetivos específicos para a área de TI. Ele estabelece as diretrizes e as metas que orientam a construção do Planejamento de TI do Órgão...”. (Brasil, 2012 p.16) 
A Reforma do Aparelho Administrativo no Brasil visou a uma melhor gestão dos recursos públicos e à implementação de políticas governamentais. Nesse sentido, Santos et al. (2006) afirmam que para que todos esses processos sejam viabilizados, a implementação dos recursos oferecidos pela TI se torna imperiosa, pois eles, por si sós, são elementos que estimulam qualidade e produtividade na gestão pública.

Na visão de Laia et al. (2011), o desenvolvimento tecnológico permitiu o crescimento nos serviços eletrônicos, a abertura da comunicação de canais com a sociedade e a divulgação das iniciativas da administração pública. Esses fatores auxiliam para a execução de ações que corroborem as necessidades de eficácia, eficiência, transparência e maior controle popular preconizados pela reforma administrativa. Da mesma forma, Carvalho (2017) também pontua que a TI mantém um papel de recurso estratégico para a administração pública. Esse fato se dá pela sua vinculação a programas de modernização da administração pública, proporcionando mais transparência e permitindo melhor governança e governabilidade.

Contudo, Hilbert e López (2011) destacam o aumento significativo dos gastos com a utilização de ferramentas digitais e aplicação do governo eletrônico, porém que ainda apresenta resultados ambíguos quanto aos reais benefícios desse aumento. Nesse sentido, os autores reforçam que, para além da aplicação das tecnologias, faz-se extremamente necessário o alinhamento entre as unidades de negócio e as unidades de tecnologia da informação.

\subsection{Alinhamento estratégico da TI}

Galas e Ponte (2005) apontam as significativas mudanças do papel da TI, dentro das organizações. O setor sai do seu papel tradicional de atividade meio, baseado apenas em prestar suporte administrativo, para uma função mais estratégica. As novas tecnologias permitiram que a TI pudesse passar a apoiar as estratégias organizacionais, viabilizando a integração entre as diversas áreas. Para tanto, o tripé estratégia de TI, estratégia empresarial e planejamento empresarial deve ter suas partes bem integradas, alinhadas e sinérgicas entre si (Rezende, 2002).

O conceito de alinhamento estratégico entre negócio e TI vem sendo abordado desde os idos de 60, 70 e 80, como demonstram Pauli e Mello Barreto (2011), nos trabalhos de Leavitt, Mclean e Soden, Rockart e Scott Morton. Entretanto, só na década de 90, Henderson e Venkatraman oferecem uma das visões mais referenciadas sobre o assunto.

O conceito de Alinhamento Estratégico da TI é muito abrangente e diversificado. Ele pode variar desde questões puramente técnicas (atuando em conceitos sobre hardware e 
software) até no que tange a modelos de gestão corporativa (Carvalho, 2017). No quadro 1, observa-se como alguns autores tratam esses aspectos.

Quadro 1 - Conceitos de alinhamento estratégico da tecnologia da informação

\begin{tabular}{|c|c|}
\hline Autores & Conceito \\
\hline KING, 1988 & $\begin{array}{l}\text { O alinhamento entre o plano estratégico de negócio e } \\
\text { plano estratégico de tecnologia de informação é } \\
\text { alcançado quando o conjunto de estratégias de sistema } \\
\text { é derivado do conjunto estratégico organizacional. }\end{array}$ \\
\hline HENDERSON, VENKATEMAN, 1993 & $\begin{array}{l}\text { O alinhamento estratégico corresponde à adequação } \\
\text { entre ambientes externo (mercado) e interno } \\
\text { (estrutura administrativa e recursos financeiros, } \\
\text { tecnológicos e humanos) e a integração funcional com } \\
\text { objetivo de desenvolver as competências e maximizar } \\
\text { o desempenho organizacional. Pode-se afirmar que é a } \\
\text { adequação entre as competências, estratégias e } \\
\text { objetivos de negócio com as de TI. }\end{array}$ \\
\hline REICH, BENBASAT, 1996 & $\begin{array}{l}\text { O elo entre o planejamento estratégico de negócios e } \\
\text { o de TI corresponde ao grau no qual a missão, os } \\
\text { objetivos e o plano de TI refletem, suporta e é } \\
\text { suportado pelos objetivos, missão e planos do } \\
\text { negócio. }\end{array}$ \\
\hline CHAN et al., 1997 & $\begin{array}{l}\text { O alinhamento entre o planejamento estratégico de } \\
\text { negócio e o de TI é a adequação da orientação } \\
\text { estratégica do negócio com a de TI. Busca-se associar } \\
\text { alinhamento estratégico de TI com a efetividade da TI } \\
\text { e o desempenho organizacional. }\end{array}$ \\
\hline
\end{tabular}

Fonte: Carvalho, 2017.

Apesar de os conceitos de planejamento estratégico terem surgido na década de 60, até 2007 tais práticas ainda não se encontravam muito disseminadas na administração pública federal. Esse fato foi constatado pelo Tribunal de Contas da União, que realizou levantamentos em 2007 e 2010 para avaliar a questão (Barros, 2014).

Tal levantamento realizado, além de apontar dados sobre o planejamento estratégico institucional, também perguntava pela área de TI e acabou por resultar no acórdão 1.603/2008Plenário que trouxe como resultado a constatação da falta de planejamento estratégico institucional e de TI, falta de planejamento das contratações de serviços de TI, falta de processo formal de planejamento de TI e falta de gente com competência adequada (Brasil, 2008).

Visando suprir tais pontos, a Secretaria de Logística e TI (SLTI) que era vinculada ao Ministério do Planejamento, exercendo a sua competência de órgão normatizador dos assuntos de TI, publicou a Instrução Normativa $n^{\circ} 04$ (IN SLTI n ${ }^{\circ}$ 04/2008), regulando as contratações de serviços de TI nos órgãos integrantes do SISP. Para o nível tático existe o Plano Diretor de Tecnologia da Informação (PDTI), que se tornou obrigatório e, para o nível estratégico, o Plano Estratégico de Tecnologia da Informação (PETI) (Barros, 2014). 
Com o passar dos anos, observou-se que, muitas vezes, o PDTI era cumprido por força da normativa, contudo acabava não existindo um PETI realmente vinculado. Isso porque, em diversos casos, a própria instituição em questão não possuía um Planejamento estratégico ao qual o PETI pudesse estar atrelado, dando o suporte tecnológico devido. Em alguns casos, como no LFO em questão, a autarquia responsável pelos diversos institutos que a compõem acabava por englobar em seu PDTI institucional as partes relativas a aquisições e serviços de seus institutos, mas nem sempre esses estavam realmente alinhados com suas estratégias internas.

Uma vez que o ente público tem o planejamento institucional definido, ele consegue trabalhar seu PETI, para que a tecnologia da informação possa atuar como um verdadeiro elemento que contribua para o sucesso da instituição. Contudo, não basta apenas que existam o PEI e o PETI; ambos devem estar alinhados e em consonância para um perfeito funcionamento de seus objetivos institucionais.

Nesse sentido, trazemos à tona o alinhamento estratégico de TI com o negócio. Apesar da existência de alguns modelos, com seus conceitos vindos desde os anos 90, a aplicabilidade vai depender do que se melhor encaixa na realidade de cada instituição. Quando tratamos de entes públicos, não teríamos realidade diferente, mesmo com peculiaridades inerentes ao setor. Sendo assim, trabalharemos em cima daqueles que acreditamos que melhor se apliquem a tal realidade.

Joia e Souza (2009) colocam que para alguns autores como Rigoni (2006) e Rezende (2001), o modelo de Henderson e Venkatraman (1993) é o modelo mais clássico e discutido da literatura de alinhamento estratégico da TI. Esse modelo preocupou-se em trazer para a formalidade os componentes estratégicos, de negócio e de TI, de forma a assegurar a semelhança dos processos (Johann et al., 2014).

O modelo de Reich e Benbasat (1996) coloca que elementos vinculados a missão, objetivos e planos de TI são articulados a missão, objetivos e planos de negócios por meio da amarração dos planejamentos de TI com os objetivos de negócios. Além disso, aponta que os níveis de entendimento da TI por parte dos executivos tem relação direta com o alinhamento (Affeldt \& Vanti, 2009).

Brodbeck e Hoppen (2003), outros autores a se observar, tinham por objetivo desenvolver um modelo operacional de alinhamento estratégico englobando os objetivos e estratégias de negócio e de tecnologia de informação, baseado na convergência de alguns elementos promotores de alinhamento dos modelos clássicos, tais quais Henderson e Venkatraman (1993) e Reich e Benbasat (1996) (Brodbeck \& Hoppen, 2003). 
Por outro lado, o modelo de Kearns e Sabherwal (2006) vem aproximar a teoria da gestão do conhecimento e o assunto alinhamento estratégico. Para tal, traz o foco para a necessidade de participação dos gerentes de negócio no planejamento estratégico de TI bem como a mão inversa, com os gerentes de TI participando do planejamento estratégico de negócio (Joia \& Souza, 2009).

Sendo assim, a proposição de um modelo híbrido composto pela adaptação dos modelos de Broadbeck e Hoppen (2003) e Kearns e Sabherwal (2006) sugere que as ideias propostas por diversos autores, durante décadas, possam ser incorporadas em seu melhor para gerar o alinhamento desejado entre negócio e tecnologia da informação. Além disso, todos os conceitos tendem a levar a um modelo que supra as necessidades comumente encontradas dentro de entes públicos: a dificuldade de comunicação entre diferentes gerências e o acompanhamento vinculante no tempo, dos planejamentos de tecnologia e de negócio, para que não andem de maneira individual.

Baseados na teoria de Kearns e Sabherwal (2006), buscamos fazer com que o DTI seja envolvido no processo de planejamento, com status de vice diretoria participante, para poder absorver melhor as necessidades das demais áreas e poder explicar melhor de que maneira pode ajudar, levando em conta a teoria de que quanto mais a alta gerência entende o operacional de TI, mais alinhados ficam os projetos.

De forma complementar, através de Broadbeck e Hoppen (2003), buscamos, com a adaptação do modelo, utilizar principalmente o alinhamento entre PETI e PEI para longo prazo com projetos de curto prazo (1 ano) sendo monitorados através de indicadores que meçam o impacto causado pela ação dentro dos Objetivos Estratégicos de TI (OETI) em cima dos OE institucionais vigentes.

A operacionalização das propostas sugeridas anteriormente seguirá a Sistemática de Desdobramento de Objetivos Estratégicos - SIDEOBES - proposta por Moreira (2002), guardadas as devidas adaptações necessárias para se adequar à realidade vivenciada pelo LFO e os objetivos de aplicação deste trabalho.

Essas adaptações, concomitantes ao uso das ferramentas do BSC aplicadas à TI e à utilização de indicadores para mensuração do desempenho obtido, vão ao encontro do que foi utilizado na confecção do planejamento estratégico vigente, bem como no desenho da cadeia de valor institucional. Tais características tendem a ser facilitadoras no momento de efetivar a comunicação de via dupla entre o negócio e a área de tecnologia. 


\section{Metodologia}

O trabalho caracteriza-se por natureza aplicada, qualitativa e objetivo exploratório, uma vez que identifica as relações entre o Planejamento Estratégico Institucional com o Planejamento Estratégico da Tecnologia da Informação.

Buscou-se montar uma proposta de modelo teórico de alinhamento estratégico com base nos pontos dos modelos de Broadbeck e Hoppen (2003) e Kearns e Sabherwal (2006). Além disso, buscaram-se também, dentro do proposto no modelo sistemático de Moreira (2002), elementos que pudessem agregar os pontos de controle e identificação de indicadores, visando a uma capacidade de ação conjunta composta por diversas fases. Moreira (2002) considera como fases um conjunto de etapas nas quais certas ações, modelos e ferramentas devem ser utilizadas para obtenção dos resultados almejados. Com isso, o autor busca uma decomposição estruturada dos objetivos estratégicos. Não nos alinharemos ao autor, em seu objetivo de desdobramento dos OE institucionais, uma vez que, no trabalho em questão, temos por objetivo o alinhamento entre OE e OETI, utilizando a ideia de sua sistemática para tal aplicação. Entretanto, de maneira análoga ao autor, também utilizaremos etapas na constituição de cada fase.

Para o levantamento dos dados contidos no planejamento estratégico institucional, a fim de extrair os objetivos estratégicos da instituição, foi necessária a análise documental do planejamento estratégico do LFO, e suas revisões, visando ao aprofundamento do que está sendo proposto como perspectivas de futuro para tal instituição. Para uma construção conjunta das necessidades institucionais, tal LFO realizou workshop que englobou os pontos: Mapa de Negócio, necessidades da partes interessadas, premissas e elementos para matriz SWOT institucional. Após aprovação em plenária das conclusões alcançadas, chegou-se aos objetivos estratégicos de maneira alinhada as perspectivas do mapa estratégico (Brasil, 2016). A partir desses levantamentos, buscaram-se subsídios para elaborar um planejamento estratégico voltado para o DTI.

Os Objetivos Estratégicos de TI (OETI) indicados dentro de cada perspectiva pontuada no Mapa estratégico proposto para o DTI foram relacionados com um OE existente na cadeia de valor Institucional vigente no LFO. Para isso, os resultados gerados pelos OETI deveriam gerar impacto em algum dos OE Institucionais. Uma segunda rodada de brainstorm entre os gestores foi realizada de modo a analisar se os OETI propostos guardavam relação com os OE institucionais. 


\section{Resultados e discussão}

O Laboratório Farmacêutico Oficial no qual desenvolveu-se este trabalho é uma organização pública de administração indireta que integra o complexo técnico-científico de uma entidade de personalidade jurídica de direito público, vinculada ao Ministério da Saúde, com sede na cidade do Rio de Janeiro (Dias, 2015).

A organização possui Planejamento Estratégico e vem procurando revê-lo periodicamente. Entretanto, nota-se um descompasso com algumas áreas internas, como a TI. $\mathrm{O}$ fato parece estar relacionado com as demandas internas se sobreporem em terem suas próprias prioridades e, embora procurem atingir a missão institucional, seguem os protocolos e procedimentos internos, não se coadunam, no total, com um Planejamento alinhado ao Planejamento macro institucional.

O confronto com a literatura bibliográfica permitiu sedimentar conceitos e propor ações para o Departamento de TI da Instituição e alta gerência, no sentido de criar um Planejamento Estratégico do Departamento alinhado com o da Instituição. Esse Planejamento define proposições e etapas para o alcance macro de forma transversal com apoio a todos os setores da Instituição, por meio da montagem de um modelo sistemático para aplicabilidade do alinhamento estratégico, conforme figura 1 .

Tomando por base as considerações de aplicabilidade apontadas na parte de Síntese e aplicabilidade dos modelos de alinhamento estratégico para TI na administração pública, buscou-se montar uma sistemática de alinhamento que pudesse conter em suas fases uma proposta híbrida contemplando pontos dos modelos de Broadbeck e Hoppen (2003) e Kearns e Sabherwal (2006). Além disso, buscaram-se também, dentro do proposto no modelo sistemático de Moreira (2002), elementos que pudessem agregar os pontos de controle e identificação de indicadores, visando a uma capacidade de ação conjunta composta por diversas fases, guardadas as devidas adaptações necessárias para se adequar à realidade vivenciada pelo LFO e os objetivos de aplicação deste trabalho.

A fase 1, denominada de Momento Pré-alinhamento, tem o objetivo de levantar os dados contidos no planejamento estratégico institucional a fim de extrair os objetivos estratégicos vigentes para a instituição. De forma semelhante ao contido na sistemática de Moreira (2002) tem-se como pressuposto que o diagnóstico estratégico institucional já foi realizado e se encontra em vigor. Como saídas desta fase temos os $\mathrm{OE}$, que, por sua vez, alimentam a fase 2 (alinhamento de longo prazo) assim como as metas institucionais para alimentar a fase 5 (controle). 
A fase 2, denominada Alinhamento de Longo Prazo, é trabalhada por Moreira (2002) para realizar a transposição dos OE em Objetivos elementares de nível Operacional (OO). Contudo, para o caso específico, essa fase será tida como base para a elaboração do planejamento estratégico de TI, tendo o institucional como referência de alinhamento. Assim sendo, utilizaremos essa fase para aplicar o entendimento advindo do modelo de Brodbeck e Hoppen (2003), voltado para o longo prazo. Buscar-se-á, através das etapas de análise ambiental e alinhamento de objetivos, a elaboração de objetivos estratégicos de TI que possam dar origem ao mapa estratégico da área ao mesmo tempo em que mantêm relações com o ambiente setorial e com a estratégia institucional. Assim, a fase 2 é composta por 2 etapas (Análise do ambiente de TI e Elaboração de OETI alinhado ao ambiente e a OE).

A fase 3 é a Identificação de indicadores baseados nos OETI elaborados que possam acompanhar a evolução/atingimento do alinhamento que será proposto. Tais indicadores obtidos serão sua saída, visando entregar ferramentas para a fase 5 (Controle).

A fase 4 é o Alinhamento de Curto Prazo e, nela, mantém-se a ideia de Broadbeck e Hoppen (2003) no que diz respeito a ações de prazo mais curto que possam ter seus resultados já impactando na estratégia institucional. Essa fase é composta por quatro etapas distintas (Acompanhamento contínuo das ações estratégicas junto aos gerentes de negócio; Alinhamento das Iniciativas Estratégicas; Elaborar ações estratégicas de TI; Implementação do Plano de Ação).

Na sistemática proposta por Moreira (2002), tal fase tem foco na operacionalização do desdobramento das estratégias priorizadas na fase anterior. Para nossa proposição, apesar de existirem etapas de operacionalização, também existirão etapas de novos alinhamentos gerenciais. Sua saída seria a realização de atividades de curto prazo, alinhadas tanto às prioridades do negócio quanto aos $\mathrm{OE}$ da instituição. Ela será o terceiro ponto de entrada para balizar a fase 5 (Controle).

A Fase 5 é a de Controle, na qual os indicadores levantados deverão ser confrontados com as metas institucionais e trarão ações e correções de planos como resultado. Seguindo os passos de Moreira (2002), ao contrário das outras fases, que são compostas apenas por uma entrada e uma saída, tal fase é composta por três entradas distintas: Metas Institucionais, Indicadores e Realizações. Ela também possui saídas múltiplas: realimentação quanto aos planos de ação (Correções) e realimentação quanto aos OETI propostos para fase 4 (Ações).

Assim como Moreira (2002), consideramos como fases um conjunto de etapas nas quais certas ações, modelos e ferramentas devem ser utilizadas para obtenção dos resultados almejados. Por outro lado, não nos alinhamos ao autor, em seu objetivo de desdobramento dos 
OE institucionais, uma vez que no trabalho em questão temos por objetivo o alinhamento entre OE e OETI, utilizando a ideia de sua sistemática para tal aplicação.

A partir da análise dos documentos, pode-se observar que, para além do planejamento estratégico, o LFO em questão caminhou em direção à gestão estratégica uma vez que foi criado um conjunto de ações que pudessem permitir aos gestores ferramentas para o cumprimento da missão e da visão institucional, atrelando em um só processo a implementação do planejamento corporativo (Brugnera et al.,2020).

Figura 1- Modelo sistemático para alinhamento

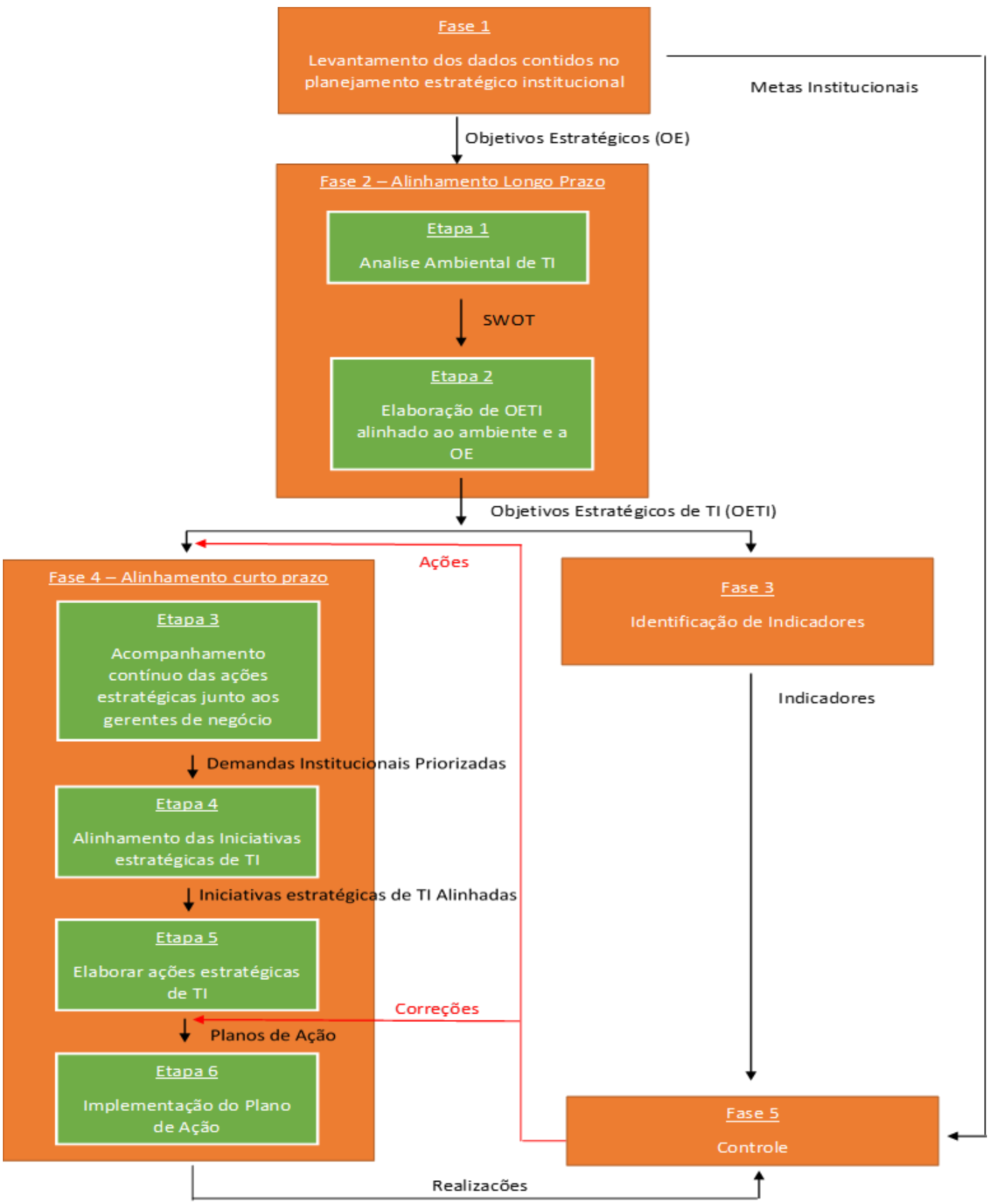

Fonte: Autor. 
Por meio do conteúdo apresentado nos documentos institucionais analisados, constatamos similaridades na aplicação das 8 (oito) etapas e atividades do processo de gerenciamento estratégico, que encontramos mencionadas em Brasil (2020): Construção da cadeia de valor; Análise Ambiental; Definição da Missão, Visão e Valores; Construção do Mapa Estratégico; Construção do Painel de Indicadores e Metas; Construção do Portfólio de Projetos e Entregas; Monitoramento intensivo da Estratégia; Monitoramento, avaliação e revisão da estratégia.

Os documentos referentes ao planejamento estratégico institucional que tomaremos como base para a construção do alinhamento desejado, nos mostram que o LFO possui 7 (sete) objetivos estratégicos institucionais que estão divididos em três perspectivas: Recursos, Processos Internos, Sociedade. Tal arranjo pode ser observado na figura 2.

Figura 2 - Perspectivas e objetivos estratégicos LFO

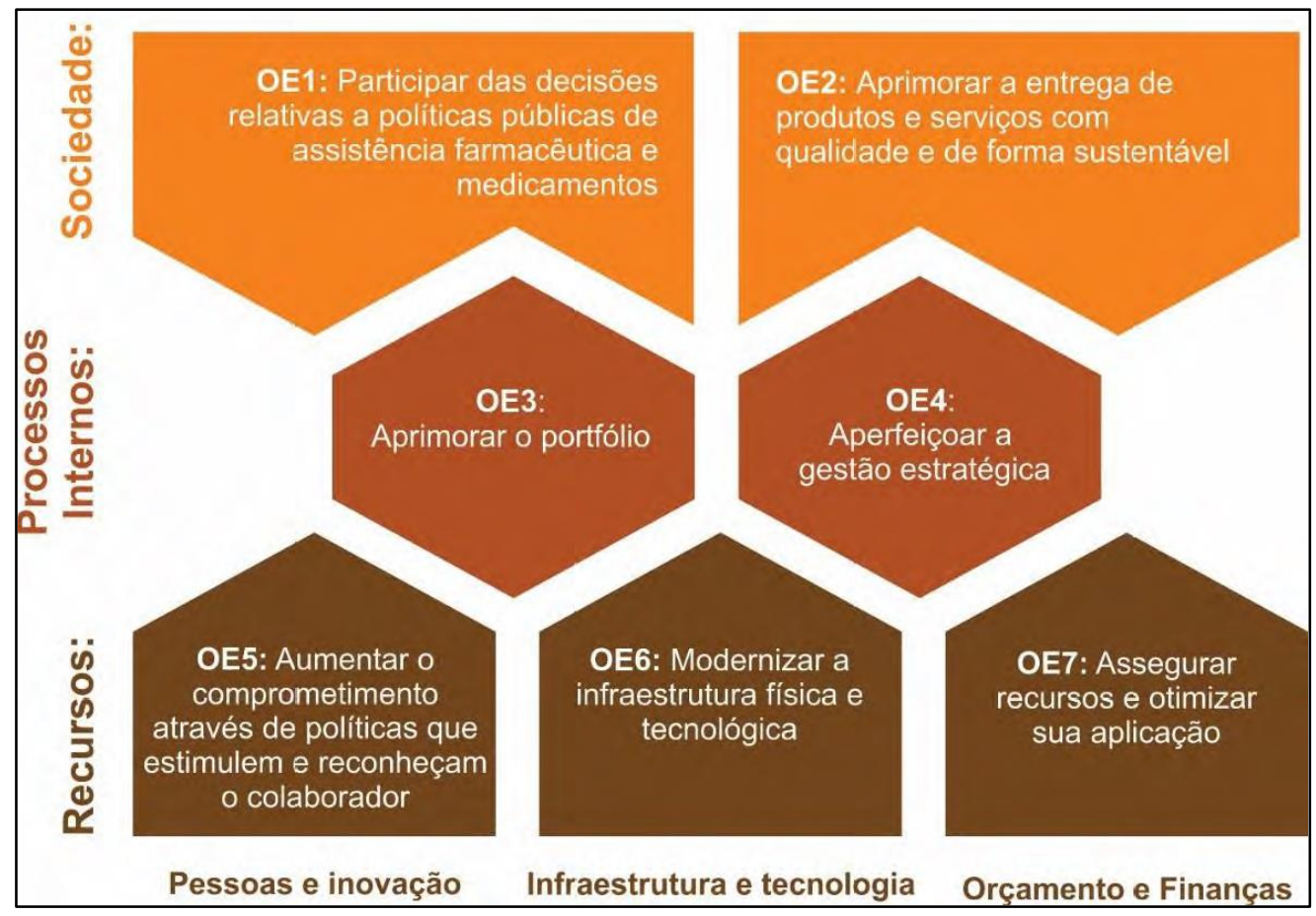

Fonte: Mapa estratégico 2016-2019 planejamento estratégico LFO.

Durante a elaboração do planejamento estratégico, realizam-se as análises de ameaças e oportunidades do ambiente externo, bem como dos pontos fortes e fragilidades do ambiente interno. Além disso, nessa etapa também são definidos os referenciais estratégicos, como: missão, visão. Apesar da divergência entre alguns autores quanto às etapas iniciais do planejamento estratégico - Paludo e Procopiuck (2011) entendem ter de iniciar pela concepção de missão e visão, enquanto outros, como Marcelino (2004) e Oliveira (2009), entendem que o 
inicio deva ser feito pelo diagnóstico organizacional - o ordenamento das etapas não prejudica

o processo de planejamento estratégico (Camargo Dias et al., 2018).

O Quadro 2 apresenta a Matriz FOFA do departamento de TI do LFO.

Quadro 2 - Matriz FOFA do departamento de TI do LFO

\begin{tabular}{|c|c|}
\hline Ambiente Interno & Ambiente Externo \\
\hline Forças & Oportunidades \\
\hline 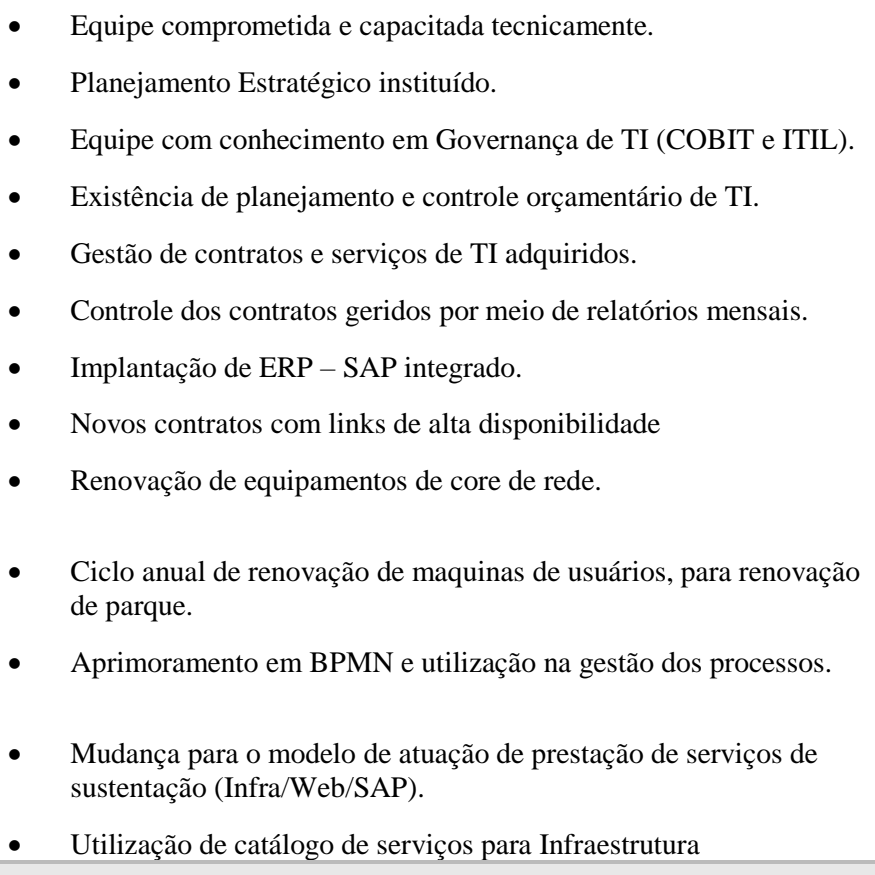 & $\begin{array}{l}\text { - } \quad \text { Utilização de consultorias externas. } \\
\text { - } \quad \text { Utilização de softwares disponibilizados pelo portal } \\
\text { softwarepublico.gov.br. } \\
\text { - } \quad \text { Utilização de novas tecnologias e frameworks de mercado. } \\
\text { - } \quad \text { Utilização de mecanismos de capacitação e desenvolvimento } \\
\text { disponibilizado pela SLTI/ENAP. } \\
\text { - } \quad \text { Expansão dos serviços e bens de TI. } \\
\text { - } \quad \text { Institucionalização de uma Política de Segurança da Informação e } \\
\text { - } \quad \text { Comunicação da FIOCRUZ. } \\
\text { Compromisso institucional na aplicação da Governança de TI. }\end{array}$ \\
\hline Fraquezas & Ameaças \\
\hline 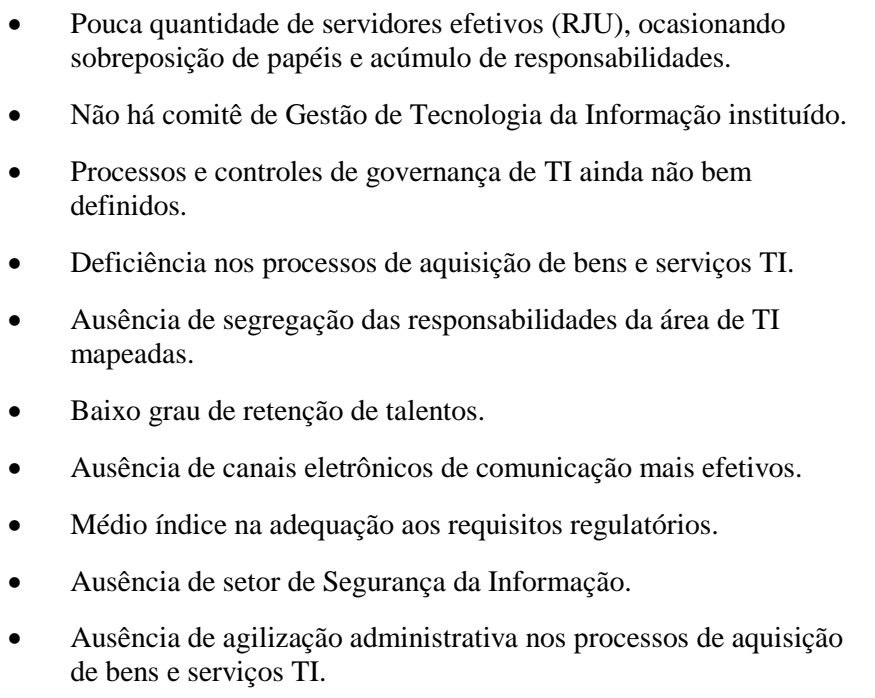 & 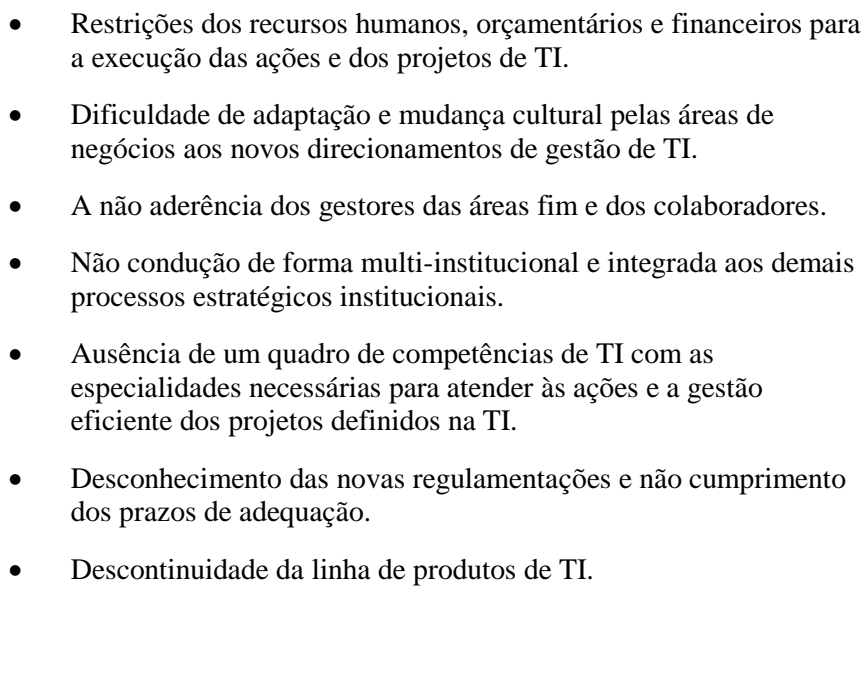 \\
\hline
\end{tabular}

Fonte: Autor. 
Para o processo de elaboração dos objetivos estratégicos de TI, também buscou-se seguir a mesma lógica utilizada para obtenção dos objetivos estratégicos institucionais, porém de maneira restrita ao departamento. Para cada perspectiva, foi elencado um conjunto de objetivos estratégicos de TI, que foram entendidos pela gerência de TI como primordiais para o alcance da missão e visão do setor.

Apesar dos OETI guardarem relação com as variáveis ambientais do setor, para que estratégias de ação possam ser melhor trabalhadas, os seus alinhamentos com os $\mathrm{OE}$ institucionais fazem-se primordiais para que a tecnologia da informação possa cumprir seu papel de suportar as necessidades corporativas no cumprimento de sua missão e alcance de sua visão. Assim sendo, os OETI também devem ser pensados de maneira sistêmica para que possam atender aos OE institucionais (Quadro 3).

\section{Quadro 3 - Perspectivas x objetivos estratégicos}

\begin{tabular}{|c|c|}
\hline Objetivos Estratégicos DTI & Objetivo Estratégico Institucional Impactado \\
\hline $\begin{array}{l}\text { OETI } 1 \text { - Prover meios eficientes de trabalho através das soluções de } \\
\text { TI }\end{array}$ & OE6 - Modernizar a infraestrutura física e tecnológica. \\
\hline \multirow[t]{2}{*}{ OETI 2 - Garantir continuidade e disponibilidade dos serviços de TI } & $\begin{array}{l}\text { OE2 - Aprimorar a entrega de produtos e serviços com } \\
\text { qualidade e de forma sustentável. }\end{array}$ \\
\hline & OE6 - Modernizar a infraestrutura física e tecnológica. \\
\hline $\begin{array}{l}\text { OETI } 3 \text { - Garantir a estrutura de TI apropriada para as atividades } \\
\text { administrativas e finalísticas }\end{array}$ & OE6 - Modernizar a infraestrutura física e tecnológica. \\
\hline OETI 4 - Aperfeiçoar Governança de TI & OE4 - Aperfeiçoar a gestão estratégica. \\
\hline OETI 5 - Aprimorar a Segurança da Informação & OE4 - Aperfeiçoar a gestão estratégica. \\
\hline $\begin{array}{l}\text { OETI } 6 \text { - Garantir desenvolvimento de competências na força de } \\
\text { trabalho de TI }\end{array}$ & $\begin{array}{l}\text { OE4 - Aperfeiçoar a gestão estratégica. } \\
\text { OE5 - Aumentar o comprometimento através de políticas que } \\
\text { estimulem e reconheçam o colaborador. }\end{array}$ \\
\hline $\begin{array}{l}\text { OETI } 7 \text { - Suportar ferramentas de TI que permitam aprimorar a } \\
\text { entrega de produtos e serviços pelo LFO }\end{array}$ & $\begin{array}{l}\text { OE2 - Aprimorar a entrega de produtos e serviços com } \\
\text { qualidade e de forma sustentável. }\end{array}$ \\
\hline $\begin{array}{l}\text { OETI } 8 \text { - Garantir a gestão e execução dos recursos orçamentários } \\
\text { de TI }\end{array}$ & OE7 - Assegurar recursos e otimizar sua aplicação. \\
\hline $\begin{array}{l}\text { OETI } 9 \text { - Prover e manter soluções de software por meio de } \\
\text { desenvolvimento ou aquisição }\end{array}$ & $\begin{array}{l}\text { OE2 - Aprimorar a entrega de produtos e serviços com } \\
\text { qualidade e de forma sustentável. } \\
\text { OE3 - Aprimorar o portfólio. } \\
\text { OE4 - Aperfeiçoar a gestão estratégica. }\end{array}$ \\
\hline
\end{tabular}

Fonte: Autor.

\section{Conclusões/considerações finais}

Os LFO são estratégicos para o Brasil como indutores de Políticas públicas e auxiliam no melhor acesso aos medicamentos para as populações menos favorecidas. Não obstante, o 
planejamento estratégico da Instituição se coaduna como qualquer outra empresa pública ou privada.

O alinhamento da estratégia institucional com todos os setores da organização é premente. Desta forma, a gestão da organização do setor de TI, alinhada com a alta gerência, promove harmonia e eficiência nos processos internos.

A existência de uma sistemática de atuação que convirja as diferentes partes entendidas como importantes para um bom alinhamento estratégico, advindas de modelos já consagrados, tende a tornar tal alinhamento mais tangível. Dessa forma, haverá mais segurança e eficácia nos processos informáticos e também maior atendimento à legislação farmacêutica vigente, bem como a diminuição de desperdícios de tempo, de retrabalho, etc.

A presença de uma fase de indicadores em tal sistemática, atrelada a uma fase de controle, poderá fornecer a curto, médio e longo prazo mecanismos para subsidiar a organização tanto no alinhamento estratégico da Instituição quanto no Departamento de Tecnologia da Informação. Tais fases ainda se encontram em desenvolvimento no presente momento.

Isso posto, é possível afirmar que o trabalho em questão contribui socialmente para todas as demais empresas ou setores públicos que desejarem aplicar o método para otimizar seus processos e práticas gerenciais e estratégicas. Até então, trata-se de uma proposta teórica que, nas próximas etapas da pesquisa de que faz parte, deverá ser validada por meio de pesquisa empírica.

Apesar de já estar em curso a aplicação do modelo sistemático proposto, faz-se necessário o monitoramento contínuo para verificações de possíveis melhorias futuras, bem como a plenitude de sua aplicabilidade, podendo este ser objeto de um novo trabalho.

\section{Referências}

Affeldt, F. S., \& Vanti, A. A. (2009). Alinhamento estratégico de tecnologia da informação: Análise de modelos e propostas para pesquisas futuras. JISTEM Journal of Information Systems and Technology Management, 6(2), 203-226. https://doi.org/10.4301/S180717752009000200004.

Anwar Aini, M., \& Suryani, E. (2019). Strategic Planning of Information System/Infromation Technology (Case Study: Gresik Regency Government Environment). IPTEK Journal of Proceedings Series, 0(5), 536. https://doi.org/10.12962/j23546026.y2019i5.6427.

Arif, F. A. (2020). IS / IT Strategic Planning of Muhammadiyah 10 GKB Senior High School. Proceedings of the 1st Annual Management, Business and Economic Conference (AMBEC 2019). 1st Annual Management, Business and Economic Conference (AMBEC 2019), Batu, Indonesia. https://doi.org/10.2991/aebmr.k.200415.006. 
Barros, F. G. (2014). Elaboração do Plano Diretor de Tecnologia da Informação (PDTI).

Bermudez, J. A. Z., Esher, A., Osorio-de-Castro, C. G. S., Vasconcelos, D. M. M. de, Chaves, G. C., Oliveira, M. A., Silva, R. M. da, \& Luiza, V. L. (2018a). Assistência Farmacêutica nos 30 anos do SUS na perspectiva da integralidade. Ciência \& Saúde Coletiva, 23(6), 1937-1949. https://doi.org/10.1590/1413-81232018236.09022018.

Bermudez, J. A. Z., Esher, A., Osorio-de-Castro, C. G. S., Vasconcelos, D. M. M. de, Chaves, G. C., Oliveira, M. A., Silva, R. M. da, \& Luiza, V. L. (2018b). Assistência Farmacêutica nos 30 anos do SUS na perspectiva da integralidade. Ciência \& Saúde Coletiva, 23(6), 1937-1949. https://doi.org/10.1590/1413-81232018236.09022018.

Brasil. (2008). Tribunal de Contas da União-Acórdão 1603/2008-Plenário. Ministro Relator: Guilherme Palmeira.

http://www.mp.go.gov.br/portalweb/hp/12/docs/acordao_tcu_-_13-08-2008.pdf\%3B.

Brasil, M. da Economia. S. E. de D., Gestão e Governo Digital. Secretaria de Gestão. (2020). Guia Técnico de Gestão Estratégica v1.0. ME; SEDGG; SEGES. https://www.gov.br/economia/pt-br/centrais-de-conteudo/publicacoes/guias-emanuais/guia_gestao_estrategica_v1-0.pdf.

Brasil, M. da S. ([s.d.]). Laboratórios Oficiais. Recuperado 9 de agosto de 2019, de http://www.saude.gov.br/assistencia-farmaceutica/laboratorios-oficiais.

Brasil, M. da Saúde. I. de T. em F. (2016). Planejamento Estratégico 2016-2019 (p. 25).

Brodbeck, Â. F., \& Hoppen, N. (2003). Alinhamento estratégico entre os planos de negócio e de tecnologia de informação: Um modelo operacional para implementação. Revista de Administração Contemporânea, 7(3), 9-33. https://doi.org/10.1590/S141565552003000300002.

Brugnera, T. L., Antunes, C. R., \& Campagnaro, R. L. (2020). REFLEXÕES SOBRE O USO DO ECONOMIC VALUE ADDED NA GESTÃO ESTRATÉGICA DE UMA COOPERATIVA DE CRÉDITO. 6(1), 13.

Camargo Dias, P. H. R. de, Sousa, J. C., \& Dias, J. C. (2018). Um estudo de caso do planejamento estratégico do IFB. Revista ibero-americana de estudos em educação, 13(1), 89-106. https://doi.org/10.21723/riaee.v13.n1.2018.9657.

Dias, A. S. da R. (2018). Plano Diretor De Tecnologia Da Informação Em Uma Organização Com Gestão De Ti Descentralizada: Uma Análise Em Unidades Técnico-Científicas Da Fiocruz [Dissertação de Mestrado, Universidade Federal da Bahia]. https://repositorio.ufba.br/ri/bitstream/ri/27594/1/Disserta\%c3\%a7\%c3\%a3o\%20de\%20Me strado\%20-\%20Andrey\%20Santana\%20da\%20Rocha\%20Dias.pdf.

Dias, E. C. F. (2015). Alinhamento da estratégia aos processos de negócios em um laboratório farmacêutico oficial: Uma proposta para Farmanguinhos. 
Falsarella, O. M., \& Jannuzzi, C. A. S. C. (2017). Planejamento Estratégico Empresarial e Planejamento de Tecnologia de Informação e Comunicação: Uma abordagem utilizando projetos. Gestão \& Produção, 24(3), 610-621. https://doi.org/10.1590/0104-530x481-16.

Figueiredo, T. A. (2015). Assistência farmacêutica no Sistema Único de Saúde e a carga de doença no Brasil. Escola Nacional de Saúde Pública.

Galas, E. S., \& Ponte, V. M. R. (2006). O Balanced Scorecard e o alinhamento estratratégico da tecnologia da informação: Um estudo de casos múltiplos. Revista Contabilidade \& FinanÂISas, 17, 37-51.

Gonçalves, C. A., Reis Neto, M. T., \& Gonçalves Filho, C. (2012). Estratégia EmpresarialO Desafio das Organizações ( $1^{\circ}$ ed). Saraiva.

Hitt, M. A., Ireland, R. D., \& Hoskisson, R. E. (2019). Administração estratégica: Competitividade e globalização $\left(12^{\circ}\right.$ ed $)$. Cengage Learning.

Johann, A. G., Johann, E. R., \& Johann, E. R. (2014). Alinhamento estratégico entre Tecnologia da Informação (TI) e o Negócio (NE): Estudo de caso no segmento de distribuição de peças de reposição de uma empresa de máquinas agrícolas. Universitas: Gestão e TI, 4(2). https://doi.org/10.5102/un.gti.v4i2.2892.

Joia, L. A., \& Souza, J. G. A. de. (2009). Articulando modelos de alinhamento estratégico de tecnologia da informação. Cadernos EBAPE.BR, 7(2), 252-269. https://doi.org/10.1590/S1679-39512009000200006.

Kaplan, R. S., \& Norton, D. P. (2006). Alinhamento: Utilizando o balanced scorecard para criar sinergias corporativas. Elsevier Brasil.

Kearns, G. S., \& Sabherwal, R. (2006). Strategic Alignment Between Business and Information Technology: A Knowledge-Based View of Behaviors, Outcome, and Consequences. Journal of Management Information Systems, 23(3), 129-162. https://doi.org/10.2753/MIS0742-1222230306.

Laia, M. M. de, Cunha, M. A. V. C. da, Nogueira, A. R. R., \& Mazzon, J. A. (2011). Electronic government policies in Brazil: Context, ICT management and outcomes. Revista de Administração de Empresas, 51(1), 43-57. https://doi.org/10.1590/S003475902011000100005 .

Magalhães, F. L. F., Gaspar, M. A., \& Campos, J. G. F. de. (2018). INFORMATION TECHNOLOGY STRATEGIC PLANNING: ANALYSIS OF CONCEPTS, FRAMEWORKS AND PROCESSES PRESENTED IN TEXTBOOKS PUBLISHED IN BRAZIL. 3065-3094. https://doi.org/10.5748/9788599693124-13CONTECSI/PS-4083.

Magalhães, J. L. D. (2010). ESTRATÉGIA GOVERNAMENTAL PARA INTERNALIZAÇÃO DE FÁRMACOS \& MEDICAMENTOS EM DOENÇAS NEGLIGENCIADAS. Universidade Federal do Rio de Janeiro. 
Margotto, J. E. S. (2019). O Laboratório Químico Farmacêutico do Exército no âmbito das Parcerias para o Desenvolvimento Produtivo. ESCOLA DE COMANDO E ESTADOMAIOR DO EXÉRCITO.

Meirelles, H. L. (2014). Direito Administrativo Brasileiro (40. ed). Malheiros Editores.

Mendes, Á., Carnut, L., \& Guerra, L. D. da S. (2018). Reflexões acerca do financiamento federal da Atenção Básica no Sistema Único de Saúde. Saúde em Debate, 42(spe1), 224243. https://doi.org/10.1590/0103-11042018s115.

Mintzberg, H., Ahlstrand, B., \& Lampel, J. (2010). Safári de Estratégia: Um Roteiro pela Selva do Planejamento Estratégico (2. ed). Bookman.

Moreira, E. (2002). Proposta de uma sistemática para o alinhamento das ações operacionais aos objetivos estratégicos, em uma gestão orientada por indicadores de desempenho. Universidade Federal de Santa Catarina.

Palmeira Filho, P. L., Pieroni, J. P., Antunes, A. M. de S., \& Martins, J. V. B. (2012). O desafio do financiamento à inovação farmacêutica no Brasil: A experiência do BNDES Profarma. http://web.bndes.gov.br/bib/jspui/handle/1408/2595.

Paranaiba, M. S. de, \& Foina, P. R. (2018). ALINHAMENTO ENTRE O PLANEJAMENTO ESTRATÉGICO E O PLANO DIRETOR DA TI EM ÓRGÃOS PÚBLICOS FEDERAIS. Universitas: Gestão e TI, 7(1). https://doi.org/10.5102/un.gti.v7i1.3763.

Pauli, S., \& Mello Barreto, L. F. B. P. (2011). Alinhamento Estratégico entre Negócios e TI. Segundo Encontro de Administração da Informação, 17.

Porter, M. E. (1986). Estratégia Competitiva: Técnicas para Análise de Indústrias e da Concorrência (7. ed). Campus.

Prahalad, C. K., \& Hamel, G. (1998). A competência essencial da corporação. In M. PORTER \& C. A. MONTGOMERY (Orgs.), Estratégia: A busca da vantagem competitiva. Campus.

Rezende, D. A. (2002). ALINHAMENTO DO PLANEJAMENTO ESTRATÉGICO DA TECNOLOGIA DA INFORMAÇÃO AO PLANEJAMENTO EMPRESARIAL: proposta de um modelo e verificação da prática em grandes empresas brasileiras [Tese, Universidade Federal de Santa Catarina]. https://repositorio.ufsc.br/xmlui/bitstream/handle/123456789/83083/PEPS2214T.pdf? sequence $=1 \&$ isAllowed $=\mathrm{y}$.

Rezende, K. S. (2013). As parcerias para o desenvolvimento produtivo e estímulo à inovação em instituições farmacêuticas públicas e privadas. As parcerias para o desenvolvimento produtivo e estímulo à inovação em instituições farmacêuticas públicas e privadas, 176.

Santos, W. J. R. dos, Riboldy, R., Fonseca, S. A., \& Bovo, J. M. (2006). A Tecnologia da Informação no Processo de Modernização Promovido pelo PMAT/BNDES no Município de São José do Rio Preto. Encontro de Administração Pública e Governança, 16. 
Silva, D. D. J. (2012). LABORATÓRIOS OFICIAIS: ESTRATÉGIAS PARA O FUNCIONAMENTO DA BAHIAFARMA. LABORATÓRIOS OFICIAIS: ESTRATÉGIAS PARA O FUNCIONAMENTO DA BAHIAFARMA, 123.

Silva, E. (2014). Complexo Econômico-Industrial da Saúde. Tempus Actas de Saúde Coletiva, 8, 71. https://doi.org/10.18569/tempus.v8i1.1455.

Souza, A. L. P. de, Pitassi, C., Bouzada, M. A. C., \& Gonçalves, A. A. (2015). A Rede Brasileira de Produção Pública de Medicamentos na perspectiva da gestão de cadeias de suprimentos: O papel das TIC. Revista de Administração Pública, 49(3), 615-641. https://doi.org/10.1590/0034-7612132157.

Teeratansirikool, L., Siengthai, S., Badir, Y., \& Charoenngam, C. (2013). Competitive strategies and firm performance: The mediating role of performance measurement. International Journal of Productivity and Performance Management, 62(2), 168-184. https://doi.org/10.1108/17410401311295722.

Ticom, P. S. P. (2019). A importância dos contratos de transferência de tecnologia nos laboratórios farmacêuticos oficiais no Brasil: O caso Farmanguinhos/FIOCRUZ. Faculdade de Ciências Humanas e Sociaisda Universidade Fernando Pessoa.

Valente, D. L., \& Brasil, C. V. de M. B. (2019). Análise de ferramentas administrativas para o planejamento estratégico. FESPPR PUBLICA, 3(1), 13.

Vasconcelos, D. M. M. de, Chaves, G. C., Azeredo, T. B., \& Silva, R. M. da. (2017). Política Nacional de Medicamentos em retrospectiva: Um balanço de (quase) 20 anos de implementação. CiÃltextordfemeninencia \& SaÃº Coletiva, 22, 2609-2614.

Zanatta, J. M. (2018). Theory of structural contingency and strategic alignment: Discussion in the theoretical field of organizational studies. 4(7), 10. 